Bull. Korean Math. Soc. 40 (2003), No. 2, pp. 177-182

\title{
FINITE METACYCLIC GROUPS WITH FAITHFUL IRREDUCIBLE REPRESENTATIONS
}

\author{
Hyo-SEOB Sim
}

\begin{abstract}
A metacyclic group is a group that has a cyclic normal subgroup with cyclic quotient. We characterize finite metacyclic groups that have faithful irreducible representations over a given field.
\end{abstract}

\section{Introduction}

A metacyclic group is a group $G$ that has a cyclic normal subgroup $K$ such that $G / K$ is also cyclic. The purpose of this paper is to characterize finite metacyclic groups which have faithful irreducible representations over a given field.

Let $G$ be a finite metacyclic group and $K$ a fixed cyclic normal subgroup such that $G / K$ is cyclic. Let $A:=\mathbf{C}_{G}(K)$ be the centralizer of $K$ in $G$. The largest normal subgroup of $G$ that is contained in a subgroup $L$ is called the core of $L$ in $G$ and is denoted by core $G L$. We say that $L$ is core-free if core $_{G} L=1$.

We shall first consider a necessary and sufficient condition for the existence of faithful irreducible representations of a finite metacyclic group $G$ in terms of the abelian normal subgroup $A$. We shall prove that the following result:

TheOREM 1.1. Let $G$ be a finite metacyclic group and let $A$ be as above. Then $G$ has a faithful irreducible representation over a field $\mathbb{F}$ if and only if the characteristic of $\mathbb{F}$ does not divide $\exp A$ and there exists a subgroup $L$ of $A$ such that $A / L$ is cyclic and core $G=1$.

The problem of characterizing the groups which have faithful irreducible representations, was initially considered in Burnside [1]; subsequently it has been treated in several papers, for example Weisner [6],

Received October 28, 2002.

2000 Mathematics Subject Classification: $20 \mathrm{C} 15$.

Key words and phrases: faithful irreducible representations, metacyclic groups. 
Kochendörffer [5], and Gaschütz [3]. The common idea involves the abelian part of the socle, that is, the product of all abelian minimal normal subgroups.

Applying the general result, we can have an alternative condition for the existence of faithful irreducible representations of metacyclic groups as follows:

TheOREM 1.2. A metacyclic group $G$ has a faithful irreducible representation over a field $\mathbb{F}$ if and only if the centre of the group $G$ is cyclic and the characteristic of $\mathbb{F}$ does not divide the order of the Fitting subgroup of $G$.

\section{Preliminaries}

We first recall some notation and terminology from representation theory. Let $\mathbb{F}$ be a field, $G$ a finite group and $H$ a subgroup of $G$. By an $\mathbb{F} G$-module we always mean a right $\mathbb{F} G$-module of finite dimension. The kernel of an $\mathbb{F} G$-module $V$ is the subgroup $\operatorname{ker} V:=\{g \in G: v g=v$ for all $v \in V\}$. We shall denote by $V \downarrow_{H}$ the $\mathbb{F} H$-module obtained by the restriction of the operators on $V$ to $\mathbb{F} H$. For an $\mathbb{F} H$-module $W$, we shall denote by $W \uparrow^{G}$ the induced $\mathbb{F} G$-module $W \otimes_{\mathbb{F} H} \mathbb{F} G$. If $H$ is normal in $G$, the conjugate of $W$ by an element $g$ in $G$ will be denoted by $W^{g}$. The subgroup $T(W):=\left\{g \in G: W^{g} \cong W\right\}$ is called the inertia group of $W$.

Let $G$ be a finite group and let $U$ be a finite $\mathbb{Z} G$-module. We shall generalize the notion of duality of vector spaces to $\mathbb{Z} G$-modules. Let $\mathbb{F}$ be a field that contains all $(\exp U)$ th roots of unity and whose characteristic does not divide the order of $U$ as a finite abelian group. Set

$$
U^{*}:=\operatorname{Hom}\left(U, \mathbb{F}^{\times}\right),
$$

where $\mathbb{F}^{\times}$is the multiplicative group of nonzero elements in $\mathbb{F}$. Then $U^{*}$ is also a $\mathbb{Z} G$-module by means of the following natural construction:

(i) if $\alpha, \beta \in U^{*}$ then define $\alpha+\beta$ by $u(\alpha+\beta)=(u \alpha)(u \beta)$ for every $u$ in $U$;

(ii) if $g \in G$ and $\alpha \in U^{*}$ then define $\alpha g$ as the map such that $u \mapsto$ $\left(u g^{-1}\right) \alpha$ for every $u$ in $U$.

The $\mathbb{Z} G$-module $U^{*}$ is called the dual of $U$. In fact, $U^{*}$ consists of all irreducible $\mathbb{F}$-representations of $U$ as an abelian group, and hence $\left|U^{*}\right|=|U|$. As a $\mathbb{Z} G$-module, $U^{* *}$ is naturally isomorphic to $U$. For 
every subgroup $V$ of $U$, define $V^{\perp}:=\left\{\alpha \in U^{*}: v \alpha=1\right.$ for all $v$ in $\left.V\right\}$. If $V$ is a submodule of the $\mathbb{Z} G$-module $U$, then $V^{\perp}$ is also a submodule of $U^{*}$. Moreover, $V^{\perp}$ is a submodule of $U^{*}$ such that $U^{*} / V^{\perp} \cong V^{*}$. Indeed, the usual relations between subspaces, quotient spaces and duality of a vector space also carry over to this case.

The prototype for such a $\mathbb{Z} G$-module in this paper is an abelian normal subgroup $A$ of $G$, regarded as a $\mathbb{Z} G$-module with respect to conjugation action. In this case, the action of $G$ on $A^{*}$ will be written in the form of $\alpha^{g}$ for $\alpha \in A^{*}$ and $g \in G$.

We prove the following lemma.

Lemma 2.1. Let $\alpha$ be an element in $U^{*}$. Then $\operatorname{core}_{G} \operatorname{ker} \alpha=1$ if and only if $\alpha$ generates $U^{*}$ as a $\mathbb{Z} G$-module.

Proof. core $_{G} \operatorname{ker} \alpha=1$ if and only if ker $\alpha$ does not contain nonzero $\mathbb{Z} G$-submodules of $U$ if and only if $\langle\alpha\rangle^{\perp}$ does not contain nonzero $\mathbb{Z} G$ submodules of $U^{* *}$ if and only if $\langle\alpha\rangle$ is not contained in a proper $\mathbb{Z} G$ submodule of $U^{*}$ if and only if $\langle\alpha\rangle$ generates $U^{*}$ as a $\mathbb{Z} G$-module.

Let $U$ be a finite semisimple $\mathbb{Z} G$-module. Then $U$ is a direct sum of simple $\mathbb{Z} G$-modules and also $U$, as an abelian group, is the direct sum of the Sylow subgroups $U_{p}$. Let $\mathbb{F}_{p}$ be the prime field of order $p$ for a prime $p$. Each simple submodule of $U_{p}$ may be regarded as an $\mathbb{F}_{p} G$-module and so $U_{p}$ is a direct sum of irreducible $\mathbb{F}_{p} G$-modules.

By Theorem VII.4.13a in [4], the multiplicity of each irreducible $\mathbb{F}_{p} G$ module $V$ as a direct summand in the largest semisimple quotient of the regular module over $\mathbb{F}_{p}$, is the dimension of $V$ over the finite field $\operatorname{End}_{\mathbb{F}_{p} G} V$. Denote the dimension by $\operatorname{Dim} V$ and call it the absolute dimension of $V$. Each $U_{p}$ is generated by a single element as a $\mathbb{F}_{p} G$-module if and only if each $U_{p}$ is a homomorphic image of the regular module $\mathbb{F}_{p} G$. Therefore, $U_{p}$ is generated by a single element if and only if for every simple $G$-module $V$, the multiplicity of $V$ in $U_{p}$ is at most $\operatorname{Dim} V$. Moreover, if $u_{p}$ generates $U_{p}$ then $\Sigma_{p} u_{p}$ also generates $\bigoplus_{p} U_{p}$, while if $u$ generates $\bigoplus_{p} U_{p}$ and $u=\Sigma_{p} u_{p}, u_{p} \in U_{p}$, then $u_{p}$ generates $U_{p}$. We then have the following fact.

Lemma 2.2. A finite semisimple $\mathbb{Z} G$-module $U$ is generated by a single element if and only if for every simple $\mathbb{Z} G$-submodule $V$, the multiplicity of $V$ as a direct summand in $U$ is at most the absolute dimension $\operatorname{Dim} V$. 


\section{Proofs of main theorems}

Let $G$ be a finite metacyclic group and $K$ a fixed cyclic normal subgroup such that $G / K$ is cyclic. Let $A:=\mathbf{C}_{G}(K)$ be the centralizer of $K$ in $G$. The group $A$ is a normal abelian subgroup of $G$ with $\mathbf{C}_{G}(A)=A$. In particular $A$ is maximal among the abelian subgroups of $G$.

We first need the following three lemmas for the proof of Theorem 1.1.

LEMma 3.1. If $L$ is a subgroup of $A$ such that $A / L$ is cyclic and core $_{G} L=1$, then $\mathbf{C}_{G}(A / L)=A$.

Proof. Since each subgroup of $K$ is characteristic in $K$ and so normal in $G$, it follows from core $G=1$ that $L \cap K=1$. Let $N$ be the normalizer of $L$. Since $N / A$ acts faithfully on $K$ and $K L / L \cong K /(K \cap L) \cong K$ are $N$-isomorphisms, the action of $N / A$ on $K L / L$ is faithful. As $K L \leq A$, it follows that $\mathbf{C}_{G}(A / L) \leq A$. The other direction of inequality is obvious, so the result follows.

Lemma 3.2. Let $\mathbb{F}$ be a field and $G$ a metacyclic group with the normal subgroup $A$ chosen as before. Let $V$ be a faithful irreducible $\mathbb{F} G$ module, let $W$ be an irreducible submodule of $V \downarrow_{A}$. Then core ${ }_{G} \operatorname{ker} W=$ 1.

Proof. Let $T$ be the inertia group of $W$. By Clifford's Theorem (see, for example, Theorem (B.7.3) in [2]), there exists an FT-module $U$ such that $U \uparrow^{G} \cong V$ and $U \downarrow_{A} \cong W^{\oplus m}$, the direct sum of $m$ copies of $W$ for some positive integer $m$. Set $L:=\operatorname{ker} U$, then $\operatorname{core}_{G} L=\operatorname{ker} V=1$. Each subgroup of $K$ is characteristic in $K$ and hence normal in $G$; we have $K \cap L=1$. Thus $[K, L]=1$, and hence $L \leq A, L=\operatorname{ker} W$. Incidentally, we have proved $\operatorname{core}_{G} \operatorname{ker} W=1$.

LEMMA 3.3. Let $\mathbb{F}$ be a field whose characteristic does not divide the order of $A$. Let $W$ be an irreducible $\mathbb{F} A$-module with core-free kernel. Then every irreducible submodule of the induced module $W^{G}$ is faithful. Conversely, each faithful irreducible $\mathbb{F} G$-module is a submodule of such a $W \uparrow^{G}$.

Proof. Let $V$ be an irreducible submodule of $W \uparrow^{G}$. Since $\left(W \uparrow^{G}\right) \downarrow_{A} \cong$ $\bigoplus W^{g}$ where the direct sum is taken over all $g$ in a transversal of $A$ in $G$, we have $V \downarrow_{A} \cong \bigoplus_{\text {some } g} W^{g}$. So $A \cap \operatorname{ker} V \leq(\operatorname{ker} W)^{g_{0}}$ for some $g_{0}$ in $G$. Because $A \cap \operatorname{ker} V$ is normal in $G$, this implies that $A \cap \operatorname{ker} V \leq$ 
$\operatorname{ker} W$. So $A \cap \operatorname{ker} V \leq \operatorname{core}_{G} \operatorname{ker} W=1$. Since $[A, \operatorname{ker} V]=1$, we have $\operatorname{ker} V \leq \mathbf{C}_{G}(A)=A$. Therefore $\operatorname{ker} V=1$.

Given a faithful irreducible $\mathbb{F} G$-module $V$, let $W$ be an irreducible constituent of the $\mathbb{F} A$-module $V \downarrow_{A}$. Since $V \downarrow_{A}$ is a sum of $G$-conjugates of $W$, we have core $G \operatorname{ker} W=1$. By the Nakayama Reciprocity (see, for example, Theorem (B.6.5) in [2]),

$$
0 \neq \operatorname{Hom}_{\mathbb{F} A}\left(V \downarrow_{A}, W\right) \cong \operatorname{Hom}_{\mathbb{F} G}\left(V, W \uparrow^{G}\right),
$$

so $W \uparrow^{G}$ has a submodule isomorphic to $V$.

Proof of Theorem 1.1. Lemma 3.1 and Lemma 3.2 prove the 'only if' part of Theorem 1.1; on the other hand, Lemma 3.3 proves the 'if' part. Thus the proof of Theorem 1.1 is complete.

We now turn to the proof of Theorem 1.2. We first state a general condition for the existence of faithful irreducible representations for groups, due to Gaschütz (see also Theorem (B.10.9) in [2]).

TheOrem 3.4. (Gaschütz [3]) Let $G$ be a finite group and $A$ the abelian part of the socle $S$ of $G$. Then $G$ has a faithful irreducible representation over a field $\mathbb{F}$ if and only if the characteristic of $\mathbb{F}$ does not divide the order of $A$ and $A$ is generated by a single element as a $\mathbb{Z} G-$ module with respect to the conjugation action.

Applying the above result, we here give a proof of Theorem 1.2.

Proof of Theorem 1.2. Since one direction of the result is well known (see Theorem VII.13.4, Theorem VIII.3.2 in [4]), by Theorem 3.4 it remains to prove that if the centre of a metacyclic group $G$ is cyclic and the condition for the field is satisfied, then the socle $U$ of $G$ is generated by a single element.

Suppose that the centre $Z$ of $G$ is cyclic and the condition for the underlying field is satisfied. Let $G=S K$ be a metacyclic factorization with a cyclic normal subgroup $K$. Suppose there are two different minimal normal subgroups $M_{1}, M_{2}$ of $G$ which are $G$-isomorphic. Since $K$ is cyclic, the subgroup $M_{1} \times M_{2}$ is not contained in $K$. Without loss of generality, we assume that $M_{2}$ is not contained in $K$; then

$$
M_{1} \cong_{G} M_{2} \cong_{G} M_{2} K / K \leq G / K .
$$

So $G$ acts trivially on $M_{1} \times M_{2}$, that is $M_{1} \times M_{2} \leq Z$, a contradiction to the assumption that $Z$ is cyclic. Consequently, no two distinct minimal normal subgroups are $G$-isomorphic. 
Let $M$ be a minimal normal subgroup in $G$. Since no distinct minimal normal subgroups are $G$-isomorphic, the multiplicity of $M$ as a direct summand of $U$ is 1 . It follows from Lemma 2.2 that $U$ is generated by a single element and hence the proof is complete.

\section{References}

[1] W. Burnside, Theory of Groups of Finite Order, Dover, New York, 1955.

[2] K. Doerk and T. Hawkes, Finite Soluble Groups, Walter de Gruyter, Berlin, 1992.

[3] W. Gaschütz, Endliche Gruppen mit treuen absolut-irreduziblen Darstellungen, Math. Nachr. 12 (1954), 253-255.

[4] B. Huppert and N. Blackburn, Finite Groups II, Springer-Verlag, Berlin, 1982.

[5] R. Kochendörffer, Über treue irreduzible Darstellungen endlicher Gruppen, Math. Nachr. 1 (1948), 25-39.

[6] L. Weisner, Condition that a finite group be multiply isomorphic with each of its irreducible representations, Amer. J. Math. 61 (1939), 709-712.

Division of Mathematical Sciences, Pukyong National University, Pusan 608-737, KOREA

E-mail: hsim@pknu.ac.kr 